\title{
On the Pólya Enumeration Theorem
}

\author{
L. G. FEL \\ Department of Civil Engineering, Technion, Haifa, Israel \\ Email:lfel@tx.technion.ac.il
}

\begin{abstract}
Simple formulas for the number of different cyclic and dihedral necklaces containing $n_{j}$ beads of the $j$-th color, $j \leq m$ and $\sum_{j=1}^{m} n_{j}=N$, are derived, using the Pólya enumeration theorem.
\end{abstract}

Keywords: permutations and cyclic invariance, cycle index, Pólya enumeration theorem

Among a vast number of counting problems one of the most popular is a necklace enumeration. A cyclic necklace is a coloring in $m$ colors of the vertices of a regular $N$--gon, where two colorings are equivalent if one can be obtained from the other by a cyclic symmetry $C_{N}$, e.g. colored beads are placed on a circle, and the circle may be rotated (without reflections). A basic enumeration problem is then: for given $m$ and $N=\sum_{j=1}^{m} n_{j}$, how many different cyclic necklaces containing $n_{j}$ beads of the $j$-th color are there. The answer follows by an application of the Pólya's theorem [1]: the number $\gamma\left(C_{N}, n^{m}\right)$ of different cyclic necklaces is the coefficient of $x_{1}^{n_{1}} \cdot \ldots \cdot x_{m}^{n_{m}}$ in the cycle index

$$
Z_{C_{N}}\left(x_{i}\right)=\frac{1}{N} \sum_{g \mid N} \phi(g) X_{g}^{N / g}, \quad X_{g}=x_{1}^{g}+\ldots+x_{m}^{g},
$$

where $\phi(g)$ denotes the Euler totient function and $n^{m}$ denotes a tuple $\left(n_{1}, \ldots, n_{m}\right)$.

Since $\gamma\left(C_{N}, n^{m}\right)$ is not available in closed form in standard and advanced textbooks [2-6] we found it worthwhile to derive this number from (1). In this article we prove that

$$
\begin{aligned}
& \gamma\left(C_{N}, n^{m}\right)=\frac{1}{N} \sum_{d \mid \Delta} \phi(d) P\left(k^{m}\right), \\
& \text { where } P\left(k^{m}\right)=\frac{\left(k_{1}+\ldots+k_{m}\right) !}{\prod_{j=1}^{m} k_{j} !}, k_{j}=\frac{n_{j}}{d},
\end{aligned}
$$

and $\Delta$ denotes a great common divisor $\operatorname{gcd} \mathrm{n}^{\wedge} \mathrm{m}$ of the tuple $n^{m}$. We denote also $k^{m}=\left(k_{1}, \ldots, k_{m}\right)$.

Note that the term $x_{1}^{n_{1}} \cdot \ldots \cdot x_{m}^{n_{m}}$ does appear only once in the multinomial series expansion (MSE) of (1) with a weight $P\left(n^{m}\right)$ when $g=1$,

$$
\begin{aligned}
& X_{1}^{N} \rightarrow P\left(n^{m}\right) x_{1}^{n_{1}} \cdot \ldots \cdot x_{m}^{n_{m}}, \\
& \text { where } N=n_{1}+\ldots+n_{m} .
\end{aligned}
$$

Show that for $g>1$ the polynomial $Z_{C_{N}}\left(x_{i}\right)$ contributes in $\gamma\left(C_{N}, n^{m}\right)$ if and only if $\Delta>1$. We prove that if $N$ is divisible by $g$ and $\Delta$ is not divisible by $g$ then the term $x_{1}^{n_{1}} \cdot \ldots \cdot x_{m}^{{ }_{m}}$ does not appear in MSE of (1).

Denote $N / g=L, 1<L<N$ and consider MSE of (1)

$$
X_{g}^{L}=\sum_{l_{i} \geq 0}^{l_{1}+\ldots+l_{m}=L} P\left(l^{m}\right) x_{1}^{g l_{1}} \cdot \ldots \cdot x_{m}^{g l_{m}}
$$

where $l^{m}$ denotes a tuple $\left(l_{1}, \ldots, l_{m}\right)$. However MSE in (4) does not contribute in $\gamma\left(C_{N}, n^{m}\right)$ since $\Delta$ is not divisible by $g$, i.e. we cannot provide such $g$ that $g l_{i}=n_{i}$ holds for all $i=1, \ldots, m$. Thus, we have reduced expression (1) by summing only over the divisors $d$ of $\Delta$,

$$
Z_{C_{N}}\left(x_{i}\right)=\frac{1}{N} \sum_{d \mid \Delta} \phi(d) X_{d}^{N / d} .
$$

Denoting $k_{j}=n_{j} / d, N / d=K=k_{1}+\ldots+k_{m}, \quad$ and considering MSE of (5) we obtain

$$
X_{d}^{K} \rightarrow P\left(k^{m}\right) x_{1}^{d k_{1}} \cdot \ldots \cdot x_{m}^{d k_{m}}=P\left(k^{m}\right) x_{1}^{{ }^{n}} \cdot \ldots \cdot x_{m}^{{ }^{n}}
$$

Combining (5) and (6) we arrive at (2).

It is easy to extend the explicit Formula (2) to the case of dihedral necklaces where two colorings are equivalent if one can be obtained from the other by a dihedral symmetry $D_{N}$, e.g. colored beads are placed on a circle, and the circle may be rotated and reflected. Start with the cycle indices [5] 


$$
\begin{aligned}
& 2 Z_{D_{N}}\left(x_{i}\right) \\
& =Z_{C_{N}}\left(x_{i}\right)+\left\{\begin{array}{c}
X_{1} X_{2}^{L}, \text { if } N=2 L+1, \\
\frac{1}{2}\left(X_{1}^{2} X_{2}^{L-1}+X_{2}^{L}\right), \text { if } N=2 L
\end{array}\right.
\end{aligned}
$$

If $N=2 L+1$ we have to distinguish two different cases.

1) There is one odd integer $n_{j}=2 a_{j}+1 \in n^{m}$, while the rest of $n_{i}$ are even, $n_{i}=2 a_{i}, L=\sum_{i=1}^{m} a_{i}$,

$$
\begin{aligned}
& \gamma\left(D_{N}, n^{m}\right)=\frac{1}{2}\left[P\left(n^{m}\right)+P\left(a^{m}\right)\right], \\
& \text { where } a^{m}=\left(a_{1}, \ldots, a_{j}, \ldots, a_{m}\right) .
\end{aligned}
$$

2) There is more than one odd integer

$$
\begin{aligned}
n_{j}=2 a_{j}+1 \in n^{m}, 1 \leq j \leq m, & \\
\gamma\left(D_{N}, n^{m}\right) & =\frac{1}{2} \gamma\left(C_{N}, n^{m}\right) .
\end{aligned}
$$

If $N=2 L$ we have to distinguish three different cases.

1) All integers $n_{j} \in n^{m}, j<m$ are even, $n_{j}=2 b_{j}$, and $L=\sum_{i=1}^{m} b_{i}, b^{m}=\left(b_{1}, \ldots, b_{m}\right)$,

$$
\begin{aligned}
& \gamma\left(D_{N}, n^{m}\right)= \\
& \frac{1}{2} \gamma\left(C_{N}, n^{m}\right)+\frac{1}{4} \sum_{q=1}^{m} P\left(b_{q}^{m}\right)+\frac{1}{4} P\left(b^{m}\right), \text { where } \\
& b_{1}^{m}=\left(b_{1}-1, b_{2}, b_{3}, \ldots, b_{m}\right), b_{2}^{m}=\left(b_{1}, b_{2}-1, b_{3}, \ldots, b_{m}\right), \\
& \ldots, b_{m}^{m}=\left(b_{1}, b_{2}, b_{3}, \ldots, b_{m}-1\right) .
\end{aligned}
$$

2) There is one pair of odd integers, $n_{j_{1}, j_{2}}=$ $2 c_{j_{1}, j_{2}}+1 \in n^{m}$, while the rest of $n_{i}$ are even, $n_{i}=2 c_{i}$,

$$
\begin{aligned}
& \gamma\left(D_{N}, n^{m}\right)=\frac{1}{2}\left[P\left(n^{m}\right)+P\left(c^{m}\right)\right], \\
& \text { where } c^{m}=\left(c_{1}, \ldots, c_{j_{1}}, \ldots, c_{j_{2}}, \ldots, c_{m}\right),
\end{aligned}
$$

and $L=1+c_{1}+\ldots+c_{j_{1}}+\ldots+c_{j_{2}}+\ldots+c_{m}$.

3) There is more than one pair of odd integers $n_{j_{1}, j_{2}}=2 c_{j_{1}, j_{2}}+1 \in n^{m}, 1 \leq j_{1}, j_{2} \leq m$,

$$
\gamma\left(D_{N}, n^{m}\right)=\frac{1}{2} \gamma\left(C_{N}, n^{m}\right) .
$$

This paper is dedicated to the memory of Yoram Zimmels. The research was partly supported by the Kamea Fellowship.

\section{REFERENCES}

[1] G. Pólya, "Kombinatorische anzahlbestimmungen für Gruppen, Graphen, und chemische Verbindungen," Acta Math., Vol. 68, pp. 145-254, 1937.

[2] F. Harary and E. M. Palmer, "Graphical enumeration," Academic Press, New York, 1973.

[3] J. J. Rotman, "An introduction to the theory of groups," Boston, Mass., Allyn and Bacon, Chapter 3, 1984.

[4] G. Polya and R. C. Read, "Combinatorial enumeration of groups, graphs, and chemical compounds," Springer, New York, 1987.

[5] F. Harary, "Graph theory," Reading, Addison-Wesley, MA, 1994.

[6] A. Kerber, "Applied finite group actions," 2nd Ed., Springer, Berlin, Chap. 3, 1999. 